\title{
Dual phase synergy enabled large elastic strains of nanoinclusions in a dislocation slip matrix composite
}

\author{
Junsong Zhang, Shijie Hao ${ }^{\mathrm{b}}$, Daqiang Jiang ${ }^{\mathrm{b}}$, Yong Huan $^{\mathrm{c}}$, Lishan Cui ${ }^{\mathrm{b}, *}$, Yinong Liu ${ }^{\mathrm{a}, *}$, \\ Yang Ren ${ }^{\mathrm{d}}$, and Hong Yang ${ }^{\mathrm{a}}$
}

\author{
${ }^{\mathrm{a}}$ School of Mechanical and Chemical Engineering, The University of Western Australia, Perth, WA 6009, \\ Australia \\ ${ }^{\mathrm{b}}$ Department of Materials Science and Engineering, China University of Petroleum-Beijing, Changping, \\ Beijing 102249, China \\ 'State Key Laboratory of Nonlinear Mechanics (LNM), Institute of Mechanics, Chinese Academy of \\ Sciences, Beijing 100190, China \\ ${ }^{\mathrm{d} X}$-ray Science Division, Argonne National Laboratory, Argonne, Illinois 60439, USA
}

*Author to whom correspondences should be addressed. E-mail: lscui@cup.edu.cn (L. Cui); yinong.liu@uwa.edu.au (Y. Liu)

\begin{abstract}
Freestanding nanomaterials (such as nanowires, nanoribbons and nanotubes) are known to exhibit ultra-large elastic strains and ultra-high strengths. However, harnessing their superior intrinsic mechanical properties in bulk composites has proven to be difficult. A recent breakthrough has overcome this difficulty by using a martensitic phase transforming matrix, in which ultra-large elastic strains approaching the theoretical limit is achieved in $\mathrm{Nb}$ nanowires embedded in the matrix. This discovery, whereas breaking a long-standing challenge, still limits our ability of harnessing the exceptional properties of nanomaterials and developing ultrahigh strength bulk materials to a narrow selection of phase transforming alloy matrices. In this study, we investigated the possibility to harness the intrinsic mechanical properties of nanoinclusions in conventional dislocation slip matrix based on a principle of synergy between the inclusion and the matrix. The small spacing between the densely populated hard and dislocation-impenetrable nanoinclusions departmentalize the plastic matrix into small domains to effectively impede dislocation motion within the matrix, inducing significant strengthening and large local elastic strains of the matrix, which in turn induced large elastic strains in the nanoinclusions. This dual phase synergy is verified in a $\mathrm{Ti}_{3} \mathrm{Sn}$ inclusions/B2-NiTi(Fe) plastic matrix model materials system. The maximum elastic strain of $\mathrm{Ti}_{3} \mathrm{Sn}$ inclusion obtained in the dislocation slip matrix is comparable to that achieved in a phase transforming matrix. This finding opens new opportunities for the development of high-strength nanocomposites.
\end{abstract}

Keywords: Elastic strain; Composite; Mechanical behavior; High-energy X-ray diffraction; Dislocation slip 


\section{Introduction}

Nanomaterials, such as nanowires, nanoribbons and nanotubes, have been shown to exhibit exceptional mechanical properties far exceeding our expectations for their bulk counterparts. These properties include ultra-large elastic strains and ultra-high strengths approaching their theoretical limits ${ }^{1-9}$. For example, a Ge nanowire was measured to have a strength of $17 \mathrm{GPa}^{5}$, a $\mathrm{Cu}$ nanowire showed an unprecedented large elastic strain of $7.2 \%{ }^{6}$, and $\mathrm{Al}_{2} \mathrm{O}_{3}$ nanowires were measured to exhibit $8.5 \%$ elastic strain and $39 \mathrm{GPa}$ fracture strength ${ }^{9}$.

Given these, much effort has been made in the past three decades by scientists in the world to design composites incorporating nanoinclusions with the intention to harness their exceptional mechanical properties in bulk forms. However, the results obtained have been disappointing $^{10}$. The intrinsic mechanical properties of nanoinclusions have not been successfully translated into those in the bulk form, e.g., elastic strains of nanoinclusions embedded in composites are typically $<1.0 \%$, much lower than their intrinsic capabilities as exhibited in freestanding forms. This situation has been dubbed the "Valley of Death" in nanocomposite design ${ }^{10}$.

Over a long period in the past, the reason for this failure has been attributed to the less-than-ideal microstructure of the composites, such as imperfect distribution and alignment of nanoinclusions, low nanoinclusion volume fraction, and poor bonding and poor load transfer at the inclusion-matrix interfaces ${ }^{10,11}$. Motivated by this understanding, intensive work have been made to overcome these microstructure imperfections, and the best achievement is probably represented by a $\mathrm{Nb}$ nanowire- $\mathrm{Cu}$ matrix composite ${ }^{12,13}$. The composite contained well dispersed and well aligned $\mathrm{Nb}$ nanowires of high aspect ratios, and strong inclusion-matrix interfacial bonding. The maximum elastic strain achieved in the Nb nanowires in this composite is $\sim 1.5 \%{ }^{12,13}$, still well below the expected $4 \sim 7 \%$ intrinsic elastic strains of the freestanding nanomaterials ${ }^{1-9}$.

A recent work has overcome this long-standing challenge and achieved ultra-large elastic strains of $6.5 \%$ in $\mathrm{Nb}$ nanowire inclusions in a NiTi shape memory alloy matrix composite. The achievement is attributed to the martensitic phase transforming matrix ${ }^{14}$. This breakthrough is enabled by a novel concept of lattice strain matching between the uniform ultra-large elastic strains (4 7\%) of the nanoinclusions and the uniform lattice distortion $(\sim 7 \%)$ of the martensitic phase transformation in the matrix ${ }^{14,15}$. This concept is schematically illustrated in Fig. 1(a). The schematic depicts a situation when the composite is deformed to $3 \%$ global strain, at which point the phase transforming matrix is partially stress-induced into martensite. The red line represents the uniform elastic strain of the nanowires deformed to $3 \%$ strain as if they were free-standing. The blue line represents the lattice distortion strain distribution of the partially transformed matrix, with $7 \%$ representing the lattice distortion strain of the martensite and $0.3 \%$ represent the elastic strain of the parent phase. The purple curve represents the expected elastic strain distribution of the nanowires in martensitic phase transforming matrix ${ }^{16}$. It is seen that within the martensite regions the nanoinclusions have 
experienced large elastic strains. This is apparently due to the matching between the lattice distortion of the martensite and the elastic strain of the nanoinclusion. This strain matching condition assures effective load transfer from the matrix to the nanoinclusions at the atomic level, as expressed in the martensite regions in the figure. Continuation of the deformation will expand the martensite regions, thus the continued increase of the total length of the elastically stretched nanowire, as depicted in Fig. 1(b) for the moment of the completion of the stress-induced martensitic transformation at $7 \%$ global strain. This new concept explicitly requires the composite matrix not to deform via dislocation plasticity ${ }^{14}$, which is at the lattice level highly non-uniform (localized) and is poorly matched with the uniform elastic lattice strains of the nanoinclusions, as schematically illustrated in Fig. 1(c). In a plastic deformation matrix, at a nominal 3\% applied strain, only 3\% of the lattice points are deformed to $100 \%$ lattice strain at each dislocation site (assuming only one dislocation slip passing at each location) and the majority of the lattice remains at the low elastic strain level. Further deformation will be realized by the increase of the number of slip sites and the multiple slips at the same site, as depicted in Fig. 1(d), but the majority of the matrix lattice still remains at low elastic strains. Whereas proving its effectiveness, this martensite strain matching concept excludes a vast range of conventional metal materials and processing options for composite design by not permitting dislocation slip as the main mechanism of deformation of the matrix.
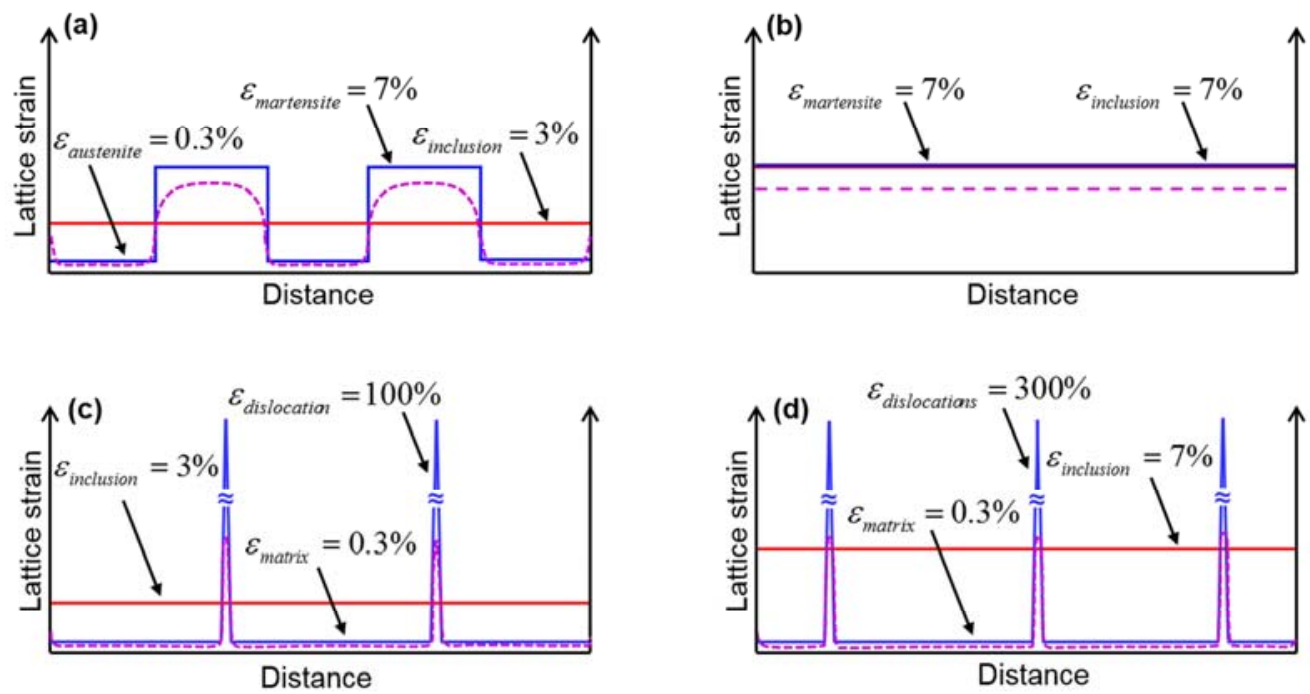

(f)
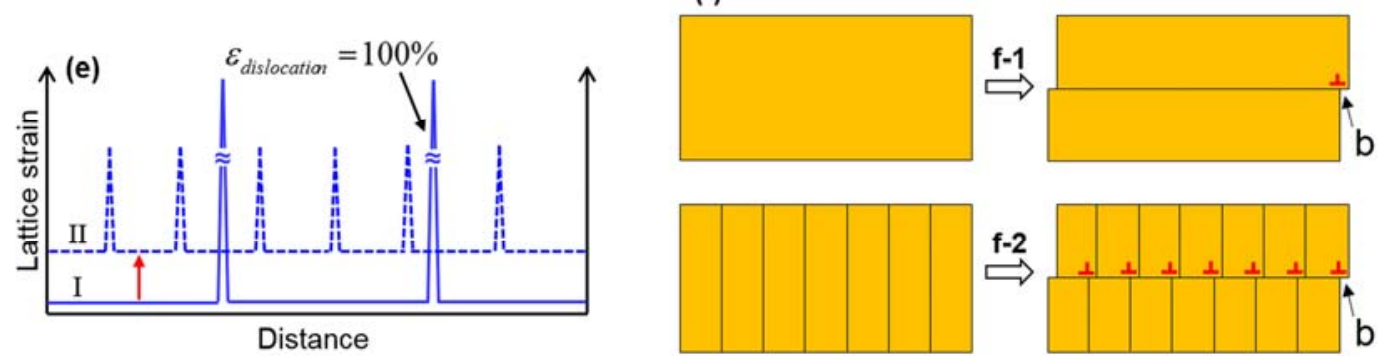

Figure 1. Schematic of the design concept. (a) and (b) are the lattice strain schematic representation of phase transforming matrix composite at 3\% global strain and $7 \%$ global strain, respectively. (c) and (d) are the lattice strain schematic representation of dislocation 
slip matrix composite at 3\% global strain and $7 \%$ global strain, respectively. The red line represents the uniform elastic strain of the nanowires deformed to 3\% strain (Fig. 1(a) and Fig. 1(c)) and 7\% strain (Fig. 1(b) and (Fig. 1(d)) as if they were free-standing. The blue line represents the lattice distortion strain distribution of the partially transformed matrix (Fig. 1(a)), the fully transformed matrix (Fig. 1(b)) and dislocation slip matrix (Fig. 1(c) and Fig. 1(d)), with 7\% representing the lattice distortion strain of the martensite (Fig. 1(a) and Fig. 1(b)), $0.3 \%$ represent the elastic strain of the parent phase (Fig. 1(a)) and dislocation slip matrix (Fig. 1(c) and Fig. 1(d)), 100\% represent the lattice distortion at each dislocation site (Fig. 1(c)) and 300\% represent the lattice distortion at dislocation pile-up site (Fig. 1(d)). The purple curve represents the expected elastic strain distribution of the nanowires in different matrices. (e) and (f) are the schematic of our design concept. Fig. 1(e) illustrates that the baseline elastic lattice strain of the matrix is increased from position I to position II. Scheme $\mathrm{f}-1$ in Fig. 1(f) represents the case of a large grain (slip domain), which experienced deformation by the passing of one dislocation. Scheme f-2 in Fig. 1(f) represents the case a large grain is departmentalized into smaller slip domains. In this case, to achieve the same magnitude of plastic deformation, a large number of dislocations are needed, with one in each domain.

\section{Design Concept}

However, rethinking of the concept of lattice strain matching, it is easy to understand that the actual reason of failure to achieve large elastic strains in the nanoinclusions in a non-martensitic matrix is not really the occurrence of dislocation slip but the low elastic lattice strain of the matrix, as illustrated in Fig. 1(c) and Fig. 1(d) for the regions between dislocations. In such matrices, the global deformation is severely localized at the lattice level at dislocation slip sites where the equivalent lattice strain can be easily several $100 \%$, resulting in the majority of the lattice experiencing minimal elastic strains well below $1 \%{ }^{4}$. It is obvious that the low elastic strains of the matrix can only induce as much elastic strains in the nanoinclusions in the composite.

Following this understanding, the strategy to overcome this problem becomes clear, i.e., to increase the baseline elastic lattice strain of the matrix from position I to position II, as schematically expressed by the dashed blue line in Fig. 1(e). This implies that the matrix needs to be significantly strengthened to be able to withstand and exhibit large elastic lattice stains, so to allow effective load transfer from the matrix to the nanoinclusions and to induce high elastic strains in them.

However, to strengthen a matrix strong enough to exhibit large lattice strains is itself a high challenge and achieving that preempts the need to develop high strength materials. The maximum elastic strain achieved in single-phase bulk nanocrystalline materials is usually only $\sim 1 \%, 17,18$. This implies that the essence of the design philosophy is to achieve dual phase synergy between the matrix and the nanoinclusions. This is possible on the basis that the matrix is expected to exhibit large lattice strains at the local level, without having to 
achieve exceptionally high global strengths. To achieve this, the nanoinclusions should departmentalize the otherwise large grains of the matrix into very small domains for dislocation slip. By doing so, the number of dislocations is drastically increased for a same nominal plastic strain compared to a large grained structure, as schematically expressed in Fig. 1(f). Scheme f-1 represents the case of a large grain (slip domain), which experienced deformation by the passing of one dislocation. Scheme $\mathrm{f}-2$ represents the case a large grain is departmentalized into smaller slip domains. In this case, to achieve the same magnitude of plastic deformation, a large number of dislocations are needed, with one in each domain. The much densely placed dislocations within the slip domains help to raise the baseline lattice strains of the matrix, as expressed by curve II in Fig. 1(e). The large lattice strains of the departmentalized matrix in turn induce large elastic strains in the nanoinclusions by the principle of lattice strain matching. In this regard, there is a clear synergy between the nanoinclusion and the matrix.

\section{Results}

To verify this hypothesis, we designed an in situ ultrafine composite composed of intermetallic $\mathrm{Ti}_{3} \mathrm{Sn}$ nanorods embedded in a $\mathrm{B} 2-\mathrm{NiTi}(\mathrm{Fe})$ plastic matrix. The matrix is designed to deform by dislocation slip instead of stress-induced martensitic transformation or martensite variant reorientation. The composite was created by eutectic solidification, and the ingot had a global composition of $\mathrm{Ti}_{57} \mathrm{Ni}_{31} \mathrm{Sn}_{8} \mathrm{Fe}_{4}$ (at. \%). Fig. 2(a) shows a scanning electron

microscope (SEM) backscattered electron image of the $\mathrm{Ti}_{3} \mathrm{Sn} / \mathrm{B} 2-\mathrm{NiTi}(\mathrm{Fe})$ composite. The composite has a complete and uniform eutectic structure with an average eutectic cell size of $6 \pm 2 \mu \mathrm{m}$. Fig. 2(b) displays the eutectic structure at a higher magnification. It consists of a continuous $\mathrm{B} 2-\mathrm{NiTi}(\mathrm{Fe})$ matrix and the $\mathrm{Ti}_{3} \mathrm{Sn}$ (bright) phase in rod form. The average rod spacing is $\sim 500 \mathrm{~nm}$ and the average rod diameter is $\sim 150 \mathrm{~nm}$. The aspect ratio of $\mathrm{Ti}_{3} \mathrm{Sn}$ rod is less than 15 . The volume fraction of $\mathrm{Ti}_{3} \mathrm{Sn}$ is estimated to be $\sim 28 \%$ by means of image analysis. Fig. 2(c) is a TEM bright-field image of the eutectic structure. Selected-area electron diffraction patterns from the matrix and the rod are shown in Fig. 2(d) and Fig. 2(e), respectively, confirming the $\mathrm{B} 2-\mathrm{NiTi}(\mathrm{Fe})$ structure and the D019- $\mathrm{Ti}_{3} \mathrm{Sn}$ structure. The composite exhibits $[-111]_{\mathrm{B} 2-\mathrm{NiTi}(\mathrm{Fe})} / /[100]_{\mathrm{D} 019-\mathrm{Ti3Sn}}$ orientation relationship between the B2-NiTi(Fe) structure and the D019- $\mathrm{Ti}_{3} \mathrm{Sn}$ structure, as shown in Fig. 2(f). EDS analysis reveals the composition of the $\mathrm{Ti}_{3} \mathrm{Sn}$ and $\mathrm{B} 2-\mathrm{NiTi}(\mathrm{Fe})$ phases in the composite to be $\mathrm{Ti}_{73.1} \mathrm{Sn}_{23.2} \mathrm{Ni}_{3.5} \mathrm{Fe}_{0.2}$ (at. \%) and $\mathrm{Ni}_{41.6} \mathrm{Ti}_{51.5} \mathrm{Fe}_{6.2} \mathrm{Sn}_{0.7}$ (at. \%), respectively. Fig. 2(g) shows a $360^{\circ}$ integrated one-dimensional (1D) $\mathrm{X}$-ray diffraction spectrum from the two-dimensional (2D) synchrotron high-energy X-ray diffraction pattern (the inset). The spectrum is fully indexed to the cubic Pm3m B2-NiTi(Fe) and the hexagonal P6 $3 / \mathrm{mmc}^{\mathrm{D} 019-\mathrm{Ti}_{3} \mathrm{Sn}}$ intermetallic phases. The inset in Fig. 2(g) is its corresponding two-dimensional high-energy $\mathrm{X}$-ray diffraction pattern of the composite. Differential scanning calorimetric measurement was also conducted, and no martensitic transformation was detected in the temperature range of $-50^{\circ} \mathrm{C} \sim 150^{\circ} \mathrm{C}$ (Fig. S1).

Fig. 2(h) is a compressive engineering stress-strain curve of $\mathrm{Ti}_{3} \mathrm{Sn} / \mathrm{B} 2-\mathrm{NiTi}(\mathrm{Fe})$ composite at 
room temperature. The inset shows its corresponding true stress-strain curve. It is seen that the composite exhibited significant strain hardening after an initial elastic deformation and achieved an ultimate compressive strength of $3.25 \mathrm{GPa}$ and a fracture strain of $45 \%$.
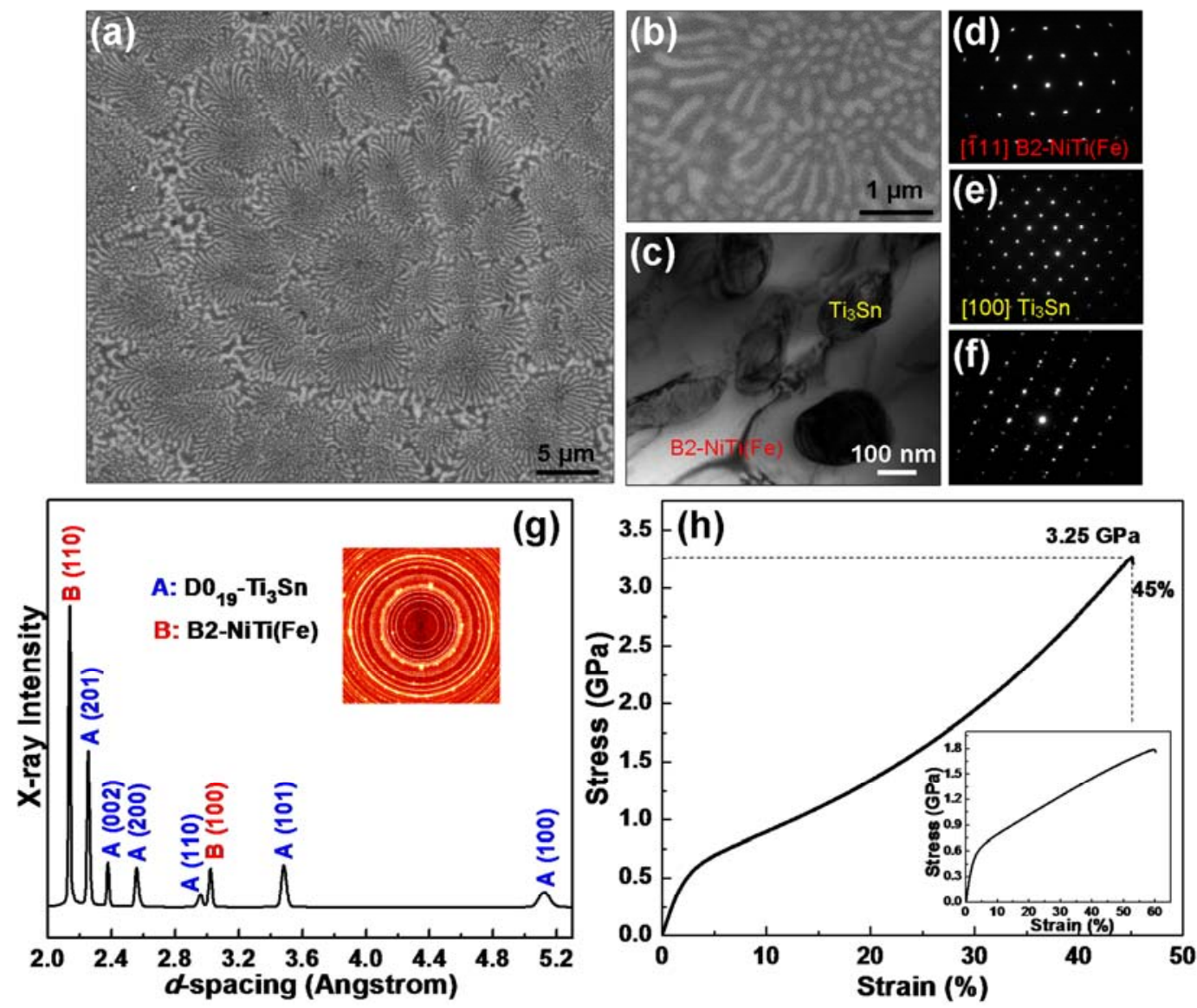

Figure 2. An in situ $\mathrm{Ti}_{3} \mathrm{Sn} / \mathrm{B} 2-\mathrm{NiTi(Fe)} \mathrm{composite.} \mathrm{(a)} \mathrm{SEM} \mathrm{backscattered} \mathrm{electron} \mathrm{image}$ of the eutectic composite. (b) $\mathrm{SEM}$ image of the $\mathrm{Ti}_{3} \mathrm{Sn}$ rod (bright) and the continuous B2-NiTi(Fe) (gray) matrix at a higher magnification. (c) TEM bright-field image of the composite. (d) and (e) display selected-area electron diffraction patterns of the B2-NiTi(Fe) matrix and $\mathrm{Ti}_{3} \mathrm{Sn}$ rod, respectively, shown in Fig. 2(c). (f) Selected-area electron diffraction pattern of the composite interface. (g) One-dimensional HE-XRD spectrum integrated at 360 degree overall of the composite. The inset contains its corresponding two-dimensional HE-XRD pattern. (h) Compressive stress-strain curve of the $\mathrm{Ti}_{3} \mathrm{Sn} / \mathrm{B} 2-\mathrm{NiTi}(\mathrm{Fe})$ composite. The inset shows its corresponding true stress-strain curve.

To uncover the microscopic mechanism of the deformation of the composite, in situ synchrotron high-energy X-ray diffraction (HE-XRD) measurements were performed during compressive deformation (Fig. S2). Fig. 3(a) shows the unrolled 2D HE-XRD pattern along the full azimuthal circle $\left(0^{\circ}\right.$ to $\left.360^{\circ}\right)$ at the maximum applied strain. The pattern is fully indexed to the cubic Pm3m B2-NiTi(Fe) and the hexagonal P6 $3 / \mathrm{mmc}$ D019-Ti 3 Sn phases, implying that the deformation did not induce any martensitic phase transformation in the B2-NiTi(Fe) matrix and that the deformation mechanism was primarily dislocation slip (Fig. 
S3). Fig. 3(b) shows the $360^{\circ}$ integrated $1 \mathrm{D}$ HE-XRD spectrums of the composite during compression. The d-spacing range within which the B19'-NiTi(Fe) diffraction peak (001), $\mathrm{d}=0.459 \mathrm{~nm}$, is expected is indicated by the dashed box, which has no overlap with other diffractions. It is evident that the B19'-NiTi(Fe) (001) diffraction peak is absent during the entire process of deformation, further proving that the B2-NiTi(Fe) matrix deformed only by dislocation slip. It is also seen that the $\mathrm{Ti}_{3} \mathrm{Sn}$ (100) diffraction peak split during deformation at high strain levels, as indicated by the twin arrows. This implies lattice strain anisotropy along the azimuthal angle, with the maximum "compression" occurring in the longitudinal direction (Fig. S4(a)) and the maximum "tension" occurring in the transverse direction (Fig. S4(b)).
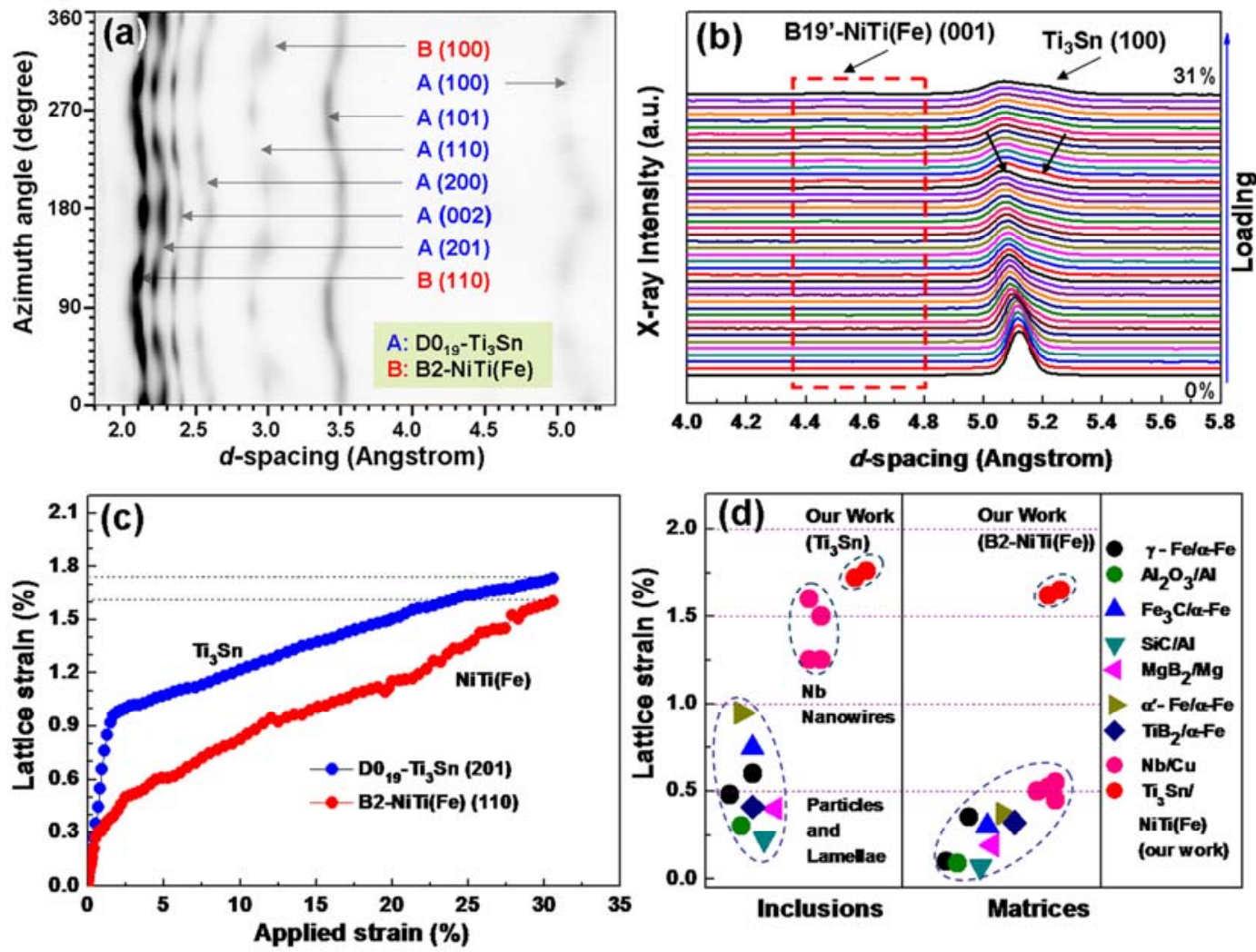

Figure 3. Microscopic deformation behavior of the $\mathrm{Ti}_{3} \mathrm{Sn} / \mathrm{B} 2-\mathrm{NiTi}(\mathrm{Fe})$ composite. (a) The unrolled 2D HE-XRD diffraction image along the azimuthal circle $\left(0^{\circ}\right.$ to $\left.360^{\circ}\right)$ at the maximum applied strain. The curved diffraction lines indicate lattice strain anisotropy in the sample. (b) Full azimuthal circle $\left(0^{\circ}\right.$ to $\left.360^{\circ}\right)$ integrated $1 \mathrm{D}$ HE-XRD spectrums of the composite during compression. (c) Evolution of B2-NiTi(Fe) (110) and $\mathrm{Ti}_{3} \mathrm{Sn}$ (201) lattice strains in the longitudinal direction as functions of the applied global strain. (d) Comparison of the elastic strains of reinforcement and matrix in the $\mathrm{Ti}_{3} \mathrm{Sn} / \mathrm{B} 2-\mathrm{NiTi}(\mathrm{Fe})$ composite with those of other metal matrix composites from the literature [black circle ${ }^{19,20}$, olive circle ${ }^{21}$, blue triangle ${ }^{22}$, dark cyan down triangle ${ }^{23}$, magenta left triangle ${ }^{24}$, dark yellow right triangle ${ }^{25}$, navy diamond ${ }^{26}$, pink circle $\left.{ }^{12,13}\right]$.

Fig. 3(c) presents the evolutions of the lattice strains of $\mathrm{Ti}_{3} \mathrm{Sn}$ (201) and B2-NiTi(Fe) (110) 
along the loading direction as functions of the applied strain. The B2-NiTi(Fe) (110) lattice strain initially increased linearly up to $0.3 \%$, reflecting the elastic deformation of the matrix, and then continued to increase at a much reduced rate with the applied strain, apparently when the plastic deformation commenced in the matrix. The maximum lattice strain reached was $1.6 \%$, at an applied global strain of $31 \%$. The strain-hardening effect, as measured by the elastic strain after the initial linear elasticity, is $1.3 \%$, or $433 \%$ of the initial linear elasticity strain. In comparison, the lattice strain of $\mathrm{Ti}_{3} \mathrm{Sn}$ (201) increased nearly linearly up to $0.95 \%$ and then continued at a much reduced rate against the applied strain to a maximum of $1.75 \%$ at $31 \%$ applied strain. B2-NiTi(Fe) is the continuous matrix which provides bulk of the inelastic deformation of the composite. $\mathrm{Ti}_{3} \mathrm{Sn}$ is the hard and discontinuous inclusion which is expected to exhibit negligible plasticity. It is also evident that the elastic lattice strains are different between $\mathrm{B} 2-\mathrm{NiTi}(\mathrm{Fe})$ and $\mathrm{Ti}_{3} \mathrm{Sn}$. This is apparently related to load partition between the more compliant $\mathrm{NiTi}(\mathrm{Fe})$ matrix and the stiffer $\mathrm{Ti}_{3} \mathrm{Sn}$ inclusions ${ }^{12,15}$.

Due to sample restriction, the maximum applied strain of the in-situ HE-XRD compression is limited to $31 \%$, which is less than the maximum strain achieved in free mechanical testing (Fig. 2(h)). From the true stress-strain curve shown in Fig. 2(h), it is seen that the strength of composite continued to increase till rupture. Thus, the maximum elastic strains reached for the two constituent phases at a global strain of $45 \%$ (60\% true strain) may be estimated by extrapolation to be $\sim 1.75 \%$ for B2-NiTi(Fe) (110) and $\sim 2.0 \%$ for $\mathrm{Ti}_{3} \mathrm{Sn}$ (201). The elastic strain of our B2-NiTi(Fe) plastic matrix is significantly higher than those found in other composite matrices that deform by dislocation slip (Fig. 3(d) $)^{12,13,19-26}$. This is the reason that the elastic strains of the $\mathrm{Ti}_{3} \mathrm{Sn}$ inclusion in our composite are much higher than those of the hard inclusions in other composites, as demonstrated in Fig. 3(d), Fig. S5(a) and Fig. $\mathrm{S} 5(\mathrm{~b})^{12,13,19-26}$. Furthermore, the maximum elastic strain of $\mathrm{Ti}_{3} \mathrm{Sn}$ achieved in our B2-NiTi(Fe) dislocation slip matrix is comparable to that achieved in the B19'-NiTi phase transforming matrix $^{15}$. A high lattice elastic strain implies high elastic stress. Using E=207 GPa for $\mathrm{Ti}_{3} \mathrm{Sn}^{27}$ and $\mathrm{E}=140 \mathrm{GPa}$ for $\mathrm{B} 2-\mathrm{NiTi}(\mathrm{Fe})^{28}$, the lattice elastic stresses, which are endured locally by the $\mathrm{Ti}_{3} \mathrm{Sn}$ nanorods and $\mathrm{B} 2-\mathrm{NiTi}(\mathrm{Fe})$ matrix in the composite, are estimated to be $4100 \mathrm{MPa}$ and $2450 \mathrm{MPa}$, respectively. This implies that the $\mathrm{Ti}_{3} \mathrm{Sn}$ is the main load bearer and significantly contributes to the strength of the composite.

\section{Discussion}

It is evident that the $\mathrm{B} 2-\mathrm{NiTi}(\mathrm{Fe})$ matrix achieved large elastic strains, reaching $1.75 \%$, and endured high elastic stresses up to be $2450 \mathrm{MPa}$. This is far greater than the strength usually measured for $\mathrm{B} 2-\mathrm{NiTi}(\mathrm{Fe})(\sim 600 \mathrm{MPa})^{28}$. At the meantime, the $\mathrm{Ti}_{3} \mathrm{Sn}$ inclusions also exhibited large elastic strains of $2.0 \%$, which is more than 6 times of what is typically measured for bulk $\mathrm{Ti}_{3} \mathrm{Sn}$ (approximately $0.3 \%$ ) $^{29}$, and high elastic stress of $4100 \mathrm{MPa}$. This is attributed to a synergistic effect between the ductile matrix and the hard inclusions.

\subsection{Effective matrix-inclusion configurations for large elastic strains}

As stated above, the central concept for increasing the baseline lattice strain is 
departmentalization of the matrix to effectively impede dislocation movement. In this regard, the microstructure of a matrix-inclusion composite may be described using three geometrical parameters: matrix grain size (g, parameter I), inclusion particle size (p, parameter II), and inclusion spacing ( $\mathrm{s}$, parameter III). Based on these three parameters, various matrix-inclusion configurations in composite can be classified into eight categories, as presented in Table S1 and schematically shown in Fig. S6. The inclusion may be in the forms of wires, ribbons, rods, and discs. For simplicity, a prolate ellipsoidal inclusion shape of two different sizes are used to represent the coarse and the fine sized inclusions.

High lattice distortion strains may be created locally when dislocations are blocked at matrix-inclusion interfaces. The elastic strain fields on either side of the interface will attenuate gradually away from the interface ${ }^{30}$. For the hard and dislocation-impenetrable inclusions (rigid body, i.e., lack of plastic flow mechanism and without dislocation channel), load, and lattice strains, need to be transferred from the matrix across the interface, thus coarse inclusions (Figs. S6(a), S6(b), S6(e) and S6(f)) are unable to achieve large mean elastic strains. This eliminates the validity of the configurations presented in Figs. S6(a), S6(b), S6(e) and S6(f) (the gray and green regions in Table S2 and Table S3). For the lattice strains in the matrix, similar argument can be made for configurations with large inter-inclusion spacings, i.e., configurations presented in Figs. S6(c) and S6(g) (the yellow and red regions in Table S2). Therefore, the only options that may have a chance to achieve large elastic strains via matrix departmentalization are those presented in Figs. S6(d) and S6(h), which are also presented in Figs. 4(b) and 4(c). These two configurations are denoted as coarse(g)/fine(p)/fine(s) and fine(g)/fine(p)/fine(s) structures. In essence, the densely spaced hard inclusions effectively departmentalize the large grains of the matrix into much smaller domains with regard to dislocation movement, and the original grain size of the matrix is less relevant. In this regard, the two configurations expressed in Figs. S6(d) and S6(h) represent similar composite conditions for the topic concerned in this discussion.

\subsection{Matrix-inclusion dual phase synergy for large elastic strains}

The synergistic effect between the matrix and the inclusions may be explained as expressed in Fig. 4. Fig. 4(a) presents the general understanding of the effect of grain size reduction on the strength (thus elastic lattice strain) of metals.

\subsubsection{Limitation of grain size reduction}

Region I in Fig. 4(a) represents the case where grain sizes are large, and the strength follows the Hall-Petch relation with grain size, as expressed by the blue line. This is due to the effect of grain boundaries in blocking the movement of dislocations, as shown in the lower inset in Region I. Continuing to decrease the grain size causes the strength to deviate from the Hall-Petch prediction, as expressed by the red curve. This is due to the increased annihilation of dislocations at the grain boundaries ${ }^{31,32}$, as schematically indicated in the lower inset in Region II. This effect becomes significant at $d<100 \mathrm{~nm}^{31,32}$. It is also known that further decreasing grain size to below 10 15 nm leads to the “inverse Hall-Petch" relation ${ }^{31,32}$ 
(Region III), in which the strength decreases with decreasing grain size, as expressed by the green curve. In this size range, the grains are incapable of generating and containing dislocations, thus can be considered rigid bodies, and plastic deformation occurs predominantly by grain boundary mediated mechanisms, such as grain sliding and grain rotation $^{31}$. In this regard, it is obvious that the ability of strength enhancement via grain size reduction is limited, and other mechanisms are needed to increase the strength in all these three regions.

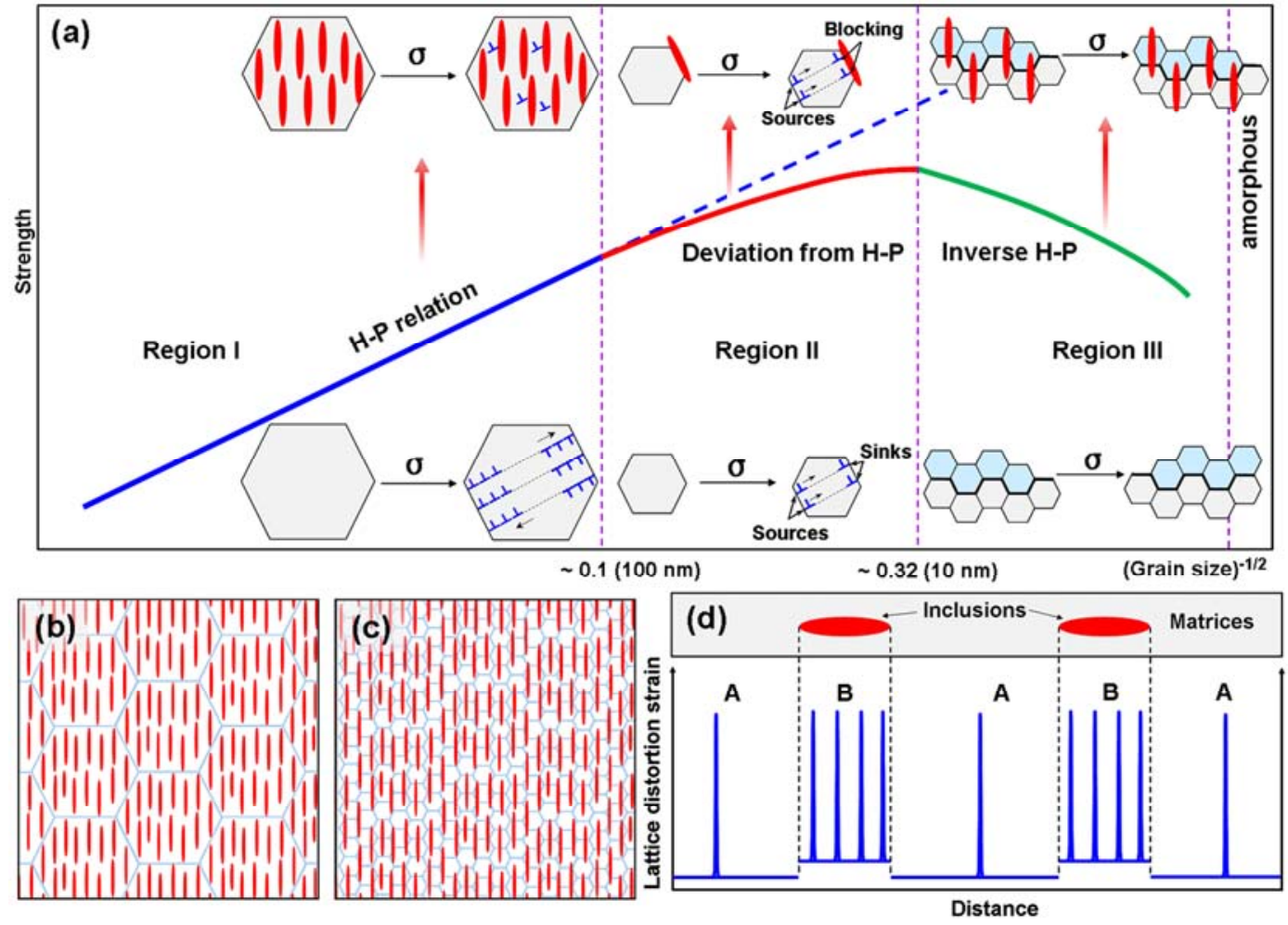

Figure 4. Schematic representation of the mechanisms to achieve large elastic strains in metal matrix composites. (a) Different mechanisms to achieve large elastic strains in different grain size regions of matrix. The solid line presents the general understanding of the effect of grain size reduction on the strength (thus elastic lattice strain) of metals. The lower insets (blue) show the deformation mechanisms in different grain size regions. The upper insets (red) show the corresponding strengthening mechanisms in the regions. Region I represents the case of conventional coarse grain. Region II $(>10 \mathrm{~nm})$ and III $(<10 \mathrm{~nm})$ represent the case of fine grains. (b) Configuration of small spacing hard nanoinclusions embedded in coarse-grained matrices. (c) Configuration of small spacing hard nanoinclusions embedded in fine-grained matrices. (d) Schematic of the higher lattice strains in the nanoinclusions than the average lattice strains of the matrix. The baseline represents the elastic lattice strain of the matrix and the peak represents the lattice distortion at dislocation sites.

\subsubsection{Strengthening in extremely fine nanograin region (Region III)}

The main limiting reason for strength in region III are the grain boundary mediated processes. Thus small and close-spaced nanoinclusions at the length scale of the grain size can pin down 
the grain boundaries to restrict their plastic activities. This concept is schematically expressed in the upper inset in Region III. This may help to eliminate the inverse Hall-Petch effect and enhance the strength to above the green curve. Recently, Hu et al. reported a similar effect of grain boundary pinning by solute segregation (solute drag) along grain boundaries to strengthen the matrix of extremely fine nanograined Ni-Mo alloy against the inverse Hall-Petch effect ${ }^{33}$. The nanoinclusion pinning strategy has the same effect to the solute drag method. Wu et al. reported a nanostructured dual-phase Mg-based alloy containing extremely fine nanocrystalline grains $(\sim 6 \mathrm{~nm})$ embedded in thin amorphous glassy matrix $(\sim 2 \mathrm{~nm}$ thick $)^{34}$. During deformation, the nanocrystalline grains block the formation and propagation of localized shear bands of the amorphous matrix, and the amorphous matrix avoid the softening effect (inverse Hall-Petch effect) due to grain boundary mediated mechanisms in traditional single-phase 6-nm-sized grained materials. This demonstrates the dual phase synergetic effect between the nanocrystalline domains and the amorphous matrix, which leads to a near-ideal strength of the Mg-based alloy.

\subsubsection{Strengthening in coarse grain region (Region I)}

In region I metals experience limited strength due to the relatively free movement of dislocations across the coarse grains. One strategy to hinder the movement of dislocations is to introduce large population of densely spaced hard nanoincluisons. These nanoinclusions effectively departmentalize the large grains into small domains for dislocation activities, akin to the effect of nanograined matrix but without the adverse effects of grain boundaries for dislocation annihilation and the inverse Hall-Petch phenomenon. This concept is

schematically illustrated in the upper inset in region I. A recent breakthrough on maraging steel clearly demonstrates this idea ${ }^{35}$. The extremely high number density and small size ( $2.7 \mathrm{~nm}$ ) of precipitates in this steel create high back stresses opposing dislocation movement, thus efficiently strengthening the matrix.

\subsubsection{Strengthening in nanograin region (Region II)}

In region II the main weakening mechanism is dislocation annihilation at grain boundaries, which requires short range movements of atoms at grain boundaries to accommodate and attenuate the lattice distortion of a dislocation ${ }^{31}$. The solution given for region I provides matrix-inclusion phase interfaces in places of grain boundaries in an otherwise nanograined matrix. The ability for a heterogeneous phase interface to accommodate and annihilate a dislocation is much lower than for a boundary between like grains because of the crystallographic incompatibility of the second phase to a dislocation. The solution given for region III has a two-fold effect on dislocation annihilation. It provides severe pinning to grain boundary activities, thus minimizing the chance of dislocation annihilation. In addition, the presence of densely spaced hard inclusions of the same size length scale of the grains also directs much of dislocation pile-ups towards these phase interfaces. As in the same argument presented above for the solution for region I, the phase boundaries have much reduced capability to annihilate dislocations. In this regard, the solutions given above for regions I and III are also effective in overcoming the main weakening mechanism in region II. 
As seen above, the solutions in all three regions effectively instigate the same strategy: to introduce densely spaced hard inclusions. These specify the structures presented in Figs. 4(b) and 4(c)) (i.e., the structures shown in Figs. S6(d) and S6(h)). In such cases, the densely spaced hard and dislocation-impenetrable nanoinclusions hinder both dislocation-instigated plasticity and grain boundary mediated plasticity to cause strengthening of the matrix, thus high elastic strains at the local lattice level, which in turn facilitate effective load transfer from the matrix to the hard nanoinclusions and induce their large elastic lattice strains. This is the dual phase synergy effect of the composite.

\subsection{Why the elastic strain of the inclusions is higher than that of the matrix}

It is also evident in Fig. 3(c) that the average lattice strain of the inclusions is always higher than that of the matrix. This is a common phenomenon also observed in similar composites (with dislocation slip matrices). It may appear anti-intuitive, because that the elastic lattice distortion of the inclusions replies on load (and lattice distortion) transfer from the matrix. This is explained as following. Upon deformation of a composite, dislocations pile up around the inclusions at the interfaces. This implies that at near the inclusions the matrix lattice distortion is much greater than the average matrix lattice strain, which is measured by XRD. On the other hand, every inclusion particle experiences such highly concentrated lattice strain levels. This situation is schematically illustrated in Fig. 4(d).

\subsection{Why earlier efforts have not worked}

Much effort has been made in the past to create super composites using nanoinclusion reinforcement in dislocation slip matrices, and none succeeded. Taking the $\mathrm{Nb}$ nanowire-Cu matrix composite for example, the maximum elastic strain of $\mathrm{Cu}$ plastic matrix achieved was $\sim 0.5 \%$ (also presented in Fig. $3(\mathrm{~d}))^{12,13}$. This is attributed to the non-rigid nature of the $\mathrm{Nb}$ nanowires, which renders them ineffective as departmentalization agent to impede dislocation motion in the $\mathrm{Cu}$ matrix, thus failed in dual phase synergy. The elastic strain limit of the $\mathrm{B} 2-\mathrm{NiTi}(\mathrm{Fe})$ plastic matrix in our composite in the initial elastic deformation stage is only $0.3 \%$, but the maximum elastic strain reached is $\sim 1.75 \%$, which is far greater than the elastic strains of free-standing $\mathrm{B} 2-\mathrm{NiTi}(\mathrm{Fe})^{28}$, or those of most other high strength bulk nanocrystalline metals or alloys (usually $\sim 1 \%)^{4,17,18}$. Obviously, such large elastic strain of the $\mathrm{B} 2-\mathrm{NiTi}(\mathrm{Fe})$ matrix is assisted by the densely populated hard $\mathrm{Ti}_{3} \mathrm{Sn}$ intermetallic inclusions.

It should be pointed out that the microstructure of the present $\mathrm{Ti}_{3} \mathrm{Sn} / \mathrm{B} 2-\mathrm{NiTi}(\mathrm{Fe})$ composite has not been optimized against the design strategy presented above. Much of structural optimization will require experimentation. Factors affecting the optimal microstructure design may include sizes and shape of the nanoinclusion particles, distribution, alignment and inter-particle spacing of the nanoinclusions, volume fraction of the nanoinclusions, and mechanical characteristics of the hard and dislocation impenetrable nanoinclusion phase. The actual values of these parameters are also dependent on the actual design objective, e.g., to achieve the best properties in Region II or Region III in Fig. 4(a). With regard to this work, 
the eutectic solidification route for the creation of the in-situ composite dictates the volume fraction of the hard $\mathrm{Ti}_{3} \mathrm{Sn}$ phase. This is a common constraint for all eutectic systems. The $\mathrm{Ti}_{3} \mathrm{Sn}$ nanorod sizes could be further refined, e.g., via more rapid solidification. The optimal nanoinclusion size and inter-particle spacing are related to the scale of multiple dislocation movement, which is required for appreciable matrix plasticity and significant strain hardening, possibly in the order of 50 100 nm (to achieve ultimate strength in the inverse Hall-Petch region the optimal nanoinclusion size and inter-particle spacing may be $\sim 10 \mathrm{~nm}$ ). Global alignment of nanorod inclusions is also desirable to maximize mechanical properties in certain directions, and this may be promoted by directional solidification. The $\mathrm{Ti}_{3} \mathrm{Sn}$ phase has relatively low elastic modulus. Based on the design concept, nanoinclusion phases of higher elastic moduli are more desired. Such optimized microstructure is expected to be able to achieve higher elastic strains and higher strengths in nanocomposites.

\section{Closing Remarks}

This work advances our understanding and ability to design high strength composite materials. It proposes a new design concept of departmentalization of plastic deforming matrix with densely spaced hard and dislocation-impenetrable nanoinclusions. The departmentalization has dual effects with synergy between to induce high strength. The densely spaced hard and dislocation-impenetrable nanoinclusions departmentalize the matrix into many small domains to effectively impede long distance dislocation slip and to force rapid dislocation multiplication during plastic deformation, causing significant strengthening and large lattice elastic strains of the matrix. The high lattice strains of the matrix in return facilitate effective load transfer from the matrix to the hard nanoinclusions and induce their large elastic lattice strains. This dual phase synergy allows harnessing the intrinsic superior mechanical properties of the nanoinclusions embedded in a plastic deforming metal matrix. This achievement significantly expands our ability to design nanoinclusion reinforced high strength composites to beyond the limitation of martensite transforming matrices as suggested previously. This concept provides a new promise and much wider options for developing high-strength nanocomposites of unprecedented mechanical properties.

\section{Methods}

A $1.5 \mathrm{~kg}$ alloy ingot with a nominal composition of $\mathrm{Ti}_{57} \mathrm{Ni}_{31} \mathrm{Sn}_{8} \mathrm{Fe}_{4}$ (at. \%) was prepared by arc melting in a water-cooled copper hearth in an argon atmosphere. Commercial purity Ti (99.99 wt. \%), Ni (99.99 wt. \%) and Sn (99.99 wt. \%) were used as raw materials. The button shaped ingot was flipped over and re-melted six times in the furnace to obtain chemical composition homogeneity. The morphology of the composite ingot was characterized using a FEI-200F scanning electron microscope (SEM) operated at $20 \mathrm{kV}$. The microstructure and chemical composition of the composite ingot were analyzed by means of a FEI Tecnai G2 F20 transmission electron microscope (TEM) equipped with an energy dispersive X-ray spectrometer (EDX) operated at accelerating voltage of $200 \mathrm{kV}$. Differential scanning calorimetry (DSC) measurement was conducted using a TA INST2910 differential scanning calorimeter with a heating/cooling rate of $10{ }^{\circ} \mathrm{C}$ $/ \mathrm{min}$. 
Mechanical properties of the composite were tested in compression using a servo-hydraulic materials testing system (MTS 810) at room temperature at a strain rate of $5 \times 10^{-4} \mathrm{~s}^{-1}$. Cylindrical compression samples of $\phi 5 \times 10 \mathrm{~mm}$ in dimension were prepared according to American Society for Testing and Materials (ASTM) standards.

In situ synchrotron high-energy X-ray diffraction (HE-XRD) measurements were performed during compression on beamline 11-ID-C at the Advanced Photon Source, Argonne National Laboratory, USA. Cylindrical samples of $\phi 1.6 \times 3.2 \mathrm{~mm}$ in dimension were prepared. High-energy X-rays with an energy level of $115 \mathrm{keV}$, wavelength of $0.10798 \AA$ and beam size of $0.6 \times 0.6 \mathrm{~mm}^{2}$ were diffracted in transmission geometry towards a Perkin-Elmer large area detector to obtain two-dimensional (2D) HE-XRD diffraction patterns. Gaussian fits were employed to determine the positions of the diffraction peaks. The lattice strain for a particular set of crystal planes is calculated as $\mid d_{\mathrm{hkl}}-d_{\mathrm{hkl}}{ }^{\circ} / d_{\mathrm{hkl}}{ }^{\circ}$, where $d_{\mathrm{hkl}}{ }^{\circ}$ is the "unstressed" lattice spacing (i.e., the peak position at zero applied stress). The error of the lattice strain measurements was estimated to be less than $0.05 \%$.

\section{Supporting Information}

Figures showing DSC result, illustration of in situ HE-XRD measurement, TEM images of the deformed composite, evolution of the diffraction peak during compression, evolution of different lattice strains as a function of the applied strain, and classification schema of departmentalization configurations for the matrix-inclusion composite. Tables showing classification of the matrix-inclusion composite and how to realize large elastic strain in conventional dislocation slip matrix composite with large spacing between inclusions and small spacing between inclusions.

\section{Acknowledgements}

This work was supported by the National Natural Science Foundation of China (NSFC) (Grant No. 51601069, 51731010, 11474362 and 51471187) and the Australian Research Council (Grant No. DP160105066 and DP180101955). The use of the Advanced Photon Source was supported by the US Department of Energy, Office of Science, and Office of Basic Energy Science, Office of Basic Energy Sciences, under Contract No. DE-AC02-06CH11357.

\section{Author Contributions}

L. Cui and Y. Liu designed the project. J. Zhang carried out materials preparation and mechanical properties testing. Y. Ren designed and supervised the synchrotron experiments. S. Hao and D. Jiang carried out the synchrotron experiments. J. Zhang carried out the SEM experiments. Y. Huan and H. Yang supervised the analysis of deformation mechanism. Y. Huan supervised the mechanical properties testing. J. Zhang and Y. Liu wrote the initial drafts of the manuscripts. J. Zhang, Y. Liu, L. Cui and H. Yang wrote the final version of the manuscript.

\section{Author Information}

The authors declare no competing financial interests. Correspondence and requests for materials should be 
addressed to L.C (lscui@cup.edu.cn) or Y.L (yinong.liu@uwa.edu.au).

\section{References}

(1) Koziol, K.; Vilatela, J.; Moisala, A.; Motta, M.; Cunniff, P.; Sennett, M.; Windle, A. Science 2007, 318, 1892-1895.

(2) Lee, C.; Wei, X.; Kysar, J. W.; Hone, J. Science 2008, 321, 385-388.

(3) Wong, E. W.; Sheehan, P. E.; Lieber, C. M. Science 1997, 277, 1971-1975.

(4) Zhu, T.; Li, J. Prog. Mater. Sci. 2010, 55, 710-757.

(5) Ngo, L. T.; Almécija, D.; Sader, J. E.; Daly, B.; Petkov, N.; Holmes, J. D.; Erts, D.; Boland, J. J. Nano Lett. 2006, 6, 2964-2968.

(6) Yue, Y.; Liu, P.; Zhang, Z.; Han, X.; Ma, E. Nano Lett. 2011, 11, 3151-3155.

(7) Richter, G.; Hillerich, K.; Gianola, D. S.; Monig, R.; Kraft, O.; Volkert, C. A. Nano Lett. 2009, 9, 3048-3052.

(8) Tian, L.; Cheng, Y. Q.; Shan, Z. W.; Li, J.; Wang, C. C.; Han, X. D.; Sun, J.; Ma, E. Nat. Commun. 2012, 3, 609.

(9) Wang, S.; He, Y.; Huang, H.; Zou, J.; Auchterlonie, G. J.; Hou, L.; Huang, B. Nanotechnology, 2013, 24, 285703.

(10) Dzenis, Y. Science, 2008, 319, 419.

(11) Podsiadlo, P.; Kaushik, A. K.; Arruda, E. M.; Waas, A. M.; Shim, B. S.; Xu, J.; Nandivada, H.; Pumplin, B. G.; Lahann, J.; Ramamoorthy, A.; Kotov, N. A. Science, 2007, 318, 80-83.

(12) Thilly, L.; Van Petegem, S.; Renault, P. O.; Lecouturier, F.; Vidal, V.; Schmitt, B.; Van Swygenhoven, H. Acta Mater. 2009, 57, 3157-3169.

(13) Vidal, V.; Thilly, L.; Van Petegem, S.; Stuhr, U.; Lecouturier, F.; Renault, P. O.; Van Swygenhoven, H. Scripta Mater. 2009, 60, 171-174.

(14) Hao, S.; Cui, L.; Jiang, D.; Han, X.; Ren, Y.; Jiang, J.; Liu, Y.; Liu, Z.; Mao, S.; Wang, Y.; Li, Y.; Ren, X.; Ding, X.; Wang, S.; Yu, C.; Shi, X.; Du, M.; Yang, F.; Zheng, Y.; Zhang, Z.; Li, X.; Brown, D. E.; Li, J. Science, 2013, 339, 1191-1194.

(15) Zhang, J.; Hao, S.; Jiang, D.; Huan, Y.; Cui, L.; Liu, Y.; Yang, H.; Ren, Y. Acta Mater. 2017, 130, 297-309.

(16) Liu, Z.; Liu, Y.; Jiang, D.; Yang, F.; Hao, S.; Ren, Y.; Cui, L. Mater. Sci. Eng. A 2014, 610, 6-9.

(17) Wang, Y.; Chen, M.; Zhou, F.; Ma, E. Nature, 2002, 419, 912-915.

(18) Lu, L.; Chen, X.; Huang, X.; Lu, K. Science, 2009, 323, 607-610.

(19) Muransky, O.; Sittner, P.; Zrník, J.; Oliver, E. C. Acta Mater. 2008, 56, 3367-3379.

(20) Jimenez-Melero, E.; Van Dijk, N. H.; Zhao, L.; Sietsma, J.; Wright, J. P.; Van der Zwaag, S. Mater. Sci. Eng. A 2011, 528, 6407-6416.

(21) Roy, S.; Gibmeier, J.; Wanner, A. Adv. Eng. Mater. 2009, 11, 471-477.

(22) Young, M. L.; Almer, J. D.; Daymond, M. R.; Haeffner, D. R.; Dunand, D. C. Acta Mater. 2007, 55, 1999-2011.

(23) Wilkes, T. E.; Harder, B. J.; Almer, J. D.; Faber, K. T. Acta Mater. 2009, 57, 6234-6242.

(24) Young, M. L.; Defouw, J.; Almer, J. D.; Dunand, D. C. Acta Mater. 2007, 55, 3467-3478.

(25) Jia, N.; Cong, Z. H.; Sun, X.; Cheng, S.; Nie, Z. H.; Ren, Y.; Liaw, P. K.; Wang, Y. D. Acta Mater. 2009, 57, 3965-3977. 
(26) Bacon, D. H.; Edwards, L.; Moffatt, J. E.; Fitzpatrick, M. E. Acta Mater. 2011, 59, 3373-3383.

(27) Catherine R, W.; Robert L, F. J. Mater. Res. 1994, 9, 1441-1448.

(28) Otsuka, K.; Ren, X. Prog. Mater. Sci. 2005, 50, 511-678.

(29) Hashimoto, T.; Nakamura, M.; Takeuchi, S. Mater. Trans. JIM 1990, 31, 195-199.

(30) Zang, K.; Mao, S.; Cai, J.; Liu, Y.; Li, H.; Hao, S.; Jiang, D.; Cui, L. Sci. Rep. 2015, 5.

(31) Meyers, M. A.; Mishra, A.; Benson, D. J. Prog. Mater. Sci. 2006, 51, 427-556.

(32) Carlton, C. E.; Ferreira, P. J. Acta Mater. 2007, 55, 3749-3756.

(33) Hu, J.; Shi, Y. N.; Sauvage, X.; Sha, G.; Lu, K. Science, 2017, 355, 1292-1296.

(34) Wu, G.; Chan, K. C.; Zhu, L.; Sun, L.; Lu, J. Nature, 2017, 545, 80-83.

(35) Jiang, S.; Wang, H.; Wu, Y.; Liu, X.; Chen, H.; Yao, M.; Gault, B.; Ponge, D.; Raabe, D.; Hirata, A.; Chen, M.; Wang, Y.; Lu, Z. Nature 2017, 544, 460-464.

\section{Table of Contents Graphic}

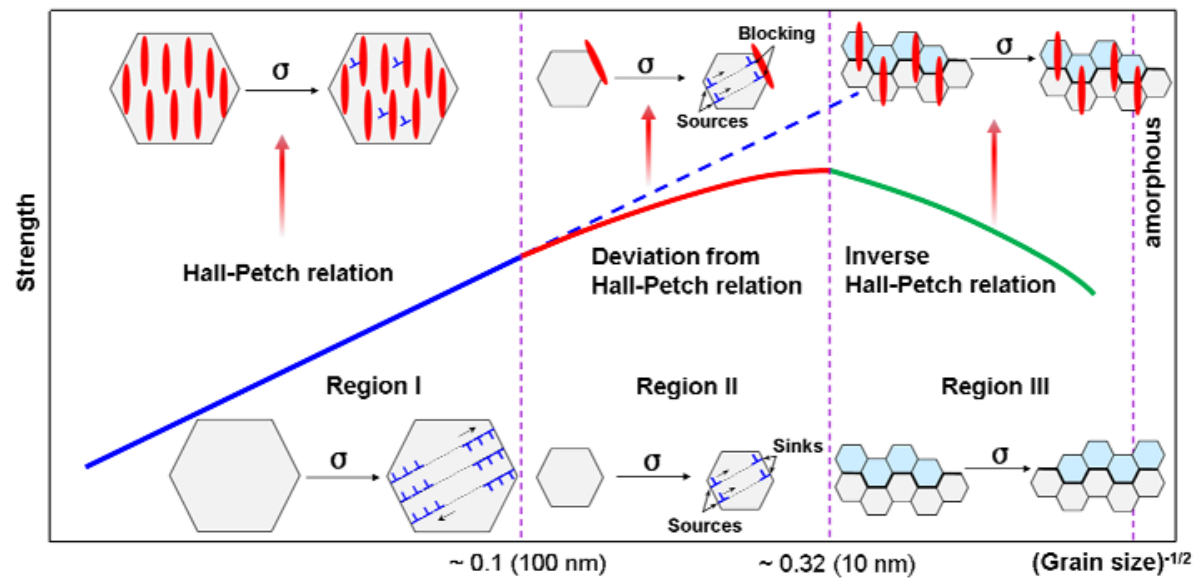

